EchoGéo

ECHOGEO Sur le Vif | 2021

\title{
Élections de 2020 aux États-Unis : des enjeux cartographiques renouvelés
}

\section{Christine Zumello}

\section{(2) OpenEdition}

Journals

Édition électronique

URL : https://journals.openedition.org/echogeo/21490

DOI : 10.4000/echogeo. 21490

ISSN : 1963-1197

\section{Éditeur}

Pôle de recherche pour l'organisation et la diffusion de l'information géographique (CNRS UMR 8586)

Référence électronique

Christine Zumello, "Élections de 2020 aux États-Unis : des enjeux cartographiques renouvelés », EchoGéo [En ligne], Sur le Vif, mis en ligne le 10 mai 2021, consulté le 24 août 2021. URL : http:// journals.openedition.org/echogeo/21490; DOI : https://doi.org/10.4000/echogeo.21490

Ce document a été généré automatiquement le 24 août 2021.

\section{(c) (i) (9)}

EchoGéo est mis à disposition selon les termes de la licence Creative Commons Attribution - Pas d'Utilisation Commerciale - Pas de Modification 4.0 International 


\title{
Élections de 2020 aux États-Unis : des enjeux cartographiques renouvelés
}

\author{
Christine Zumello
}

\section{Introduction}

1 Les élections présidentielles américaines de l'automne 2020 ont permis de voir, et revoir, de nombreuses cartes. La cartographie nationale des résultats par état est frappante. Elle est invariablement binaire: une association de bleu et de rouge. Les États remportés par les Démocrates sont bleus, les États remportés par les Républicains sont rouges, sans nuance, semble-t-il, puisque c'est le système du winner takes all. Le candidat vainqueur de l'État fédéré remporte tous les Grands Électeurs, ce qui justifie cette cartographie binaire. Or cette uniformité est trompeuse. Quelle signification accorder à cette binarité, de façade pourrait-on dire, par rapport aux incertitudes qui avaient plané sur toute la campagne électorale ? En effet, l'issue du scrutin a fait l'objet d'innombrables conjectures et d'interrogations multiples. Donald Trump allait-il être réélu pour un deuxième mandat? Les sondages légèrement favorables au candidat démocrate, Joe Biden, allaient-ils être démentis comme en 2016 où la Démocrate Hillary Clinton avait été donnée gagnante? En pleine pandémie de Covid-19, le vote par correspondance utilisé massivement allait-il être source inévitable de fraudes et de chaos comme Donald Trump le laissait entendre depuis l'été 2020 ?

2 À toutes ces questions qui planaient sur le scrutin du 3 novembre 2020 s'ajoutaient quelques certitudes. L'élection présidentielle se jouerait dans quelques États « pivots » (swing states) susceptibles de basculer dans le camp démocrate ou dans le camp républicain. En somme, l'élection fédérale se dénouerait, sur la carte, dans une poignée d'États : au nord-est, la Pennsylvanie; dans le nord du Midwest, le Michigan, le Wisconsin et l'Ohio ; au Sud, la Virginie, la Caroline du Nord, la Géorgie et la Floride, au sud-ouest, le Nevada et l'Arizona. Ces États pouvaient donc faire basculer le pays dans 
un camp ou dans l'autre. Au soir de l'élection, le 3 novembre, le suspense s'installe effectivement. La carte des résultats, selon les termes de Dan Balz du Washington Post, s'affiche telle " une mer d'indécision » à mesure que les bureaux de vote commencent le dépouillement des bulletins ${ }^{1}$. Comment réconcilier cette indécision et, pourtant, le résultat si tranché que figurent les cartes des résultats des présidentielles?

Nous examinerons ici, tout d'abord, les enjeux de la cartographie des résultats de l'élection présidentielle. Un certain nombre de choix sont opérés pour traduire les effets du scrutin uninominal à un tour et le fonctionnement du Collège Électoral. Mais, nous le verrons, d'autres choix cartographiques pourraient être faits. Puis, nous reviendrons sur les effets d'échelle de l'élection présidentielle. Si elle permet de désigner un vainqueur à une fonction nationale et fédérale, nous constaterons qu'une focale plus fine permet de nuancer les résultats de façon saisissante si l'on descend à l'échelon de l'état fédéré ou, plus local encore, celui du comté. Ces cartes plus précises, plus granulaires, traduisent, en fait, une grande complexité du tissu électoral aux ÉtatsUnis. Enfin, nous évoquerons des élections tout aussi importantes et qui, pourtant, sont souvent remisées au second plan lors des échéances présidentielles : les élections au Congrès à Washington (Chambre des représentants plus particulièrement). Là encore, ce sont des cartes des circonscriptions électorales que l'on peut tirer de nombreux enseignements sur la vigueur de la démocratie américaine. Une brève perspective historique sur les manipulations des cartes des circonscriptions permettra d'éclairer les derniers résultats (automne 2020) et d'envisager quelques pistes de réflexion et d'attention pour l'avenir.

\section{Résultats de l'élection présidentielle : plusieurs cartographies}

\section{États, collège électoral et vote populaire}

4 La carte officielle des résultats (illustration 1) montre un partage net entre deux couleurs : le bleu pour les États remportés par le Démocrate Joe Biden et le rouge pour les États remportés par le Républicain Donald Trump. On constate que les États du Midwest et du Sud à l'exception de la Géorgie sont remportés par le candidat républicain. Les États du nord du Midwest, des côtes Pacifique et Atlantique sont remportés par le candidat démocrate. Cette carte rend compte du résultat du vote au sein du Collège Électoral (232 grands électeurs pour Donald Trump et 306 grands électeurs pour Joe Biden). Une autre carte (illustration 2) montre, elle, le même résultat mais cette fois en prenant en compte le nombre de voix au sein du Collège Électoral, par État. Cette carte montre beaucoup mieux le poids de chacun des États au sein du Collège Électoral. C'est, cette fois, le nombre de grands électeurs par État qui est mis en évidence et on voit que la proportion de rouge est bien plus faible que sur l'illustration 1. En effet, l'État de New York compte 19,3 millions d'habitants pour 29 Grands Électeurs. Chacun d'entre eux représente donc 655000 newyorkais. En revanche, pour le Vermont ou le Wyoming on voit que la proportion est bien différente. Pour 623000 habitants, le Vermont a droit, lui, à 3 Grands Électeurs (le minimum incompressible) ce qui aboutit à un Grand Électeur pour 207000 habitants, soit près de trois fois moins que pour l'État de New York. De même, pour le Wyoming, avec ses 582000 habitants, chaque Grand Électeur ne représente que 194000 habitants. 
L'illustration 3 est un cartogramme qui met en relief la population des différents États. Les contours de la carte, on le voit, en sont transformés. Le centre (Midwest) est écrasé au profit des États très peuplés que l'on trouve à l'est (New York, Pennsylvanie), à l'ouest (Californie), au centre (Illinois) et au sud (Floride et Texas). C'est grâce à cette carte que l'on comprend le résultat du vote populaire : $51,38 \%$ (soit un peu plus de 80 millions de voix) pour Joe Biden contre $46,91 \%$ (soit un peu plus de 74 millions de voix) pour Donald Trump. Nous sommes, en 2020, face à un taux de participation record aux États-Unis, où la participation électorale est structurellement faible depuis plus de $50 \mathrm{ans}^{2}$. Ces données plaideraient pour que l'on affiche plus fréquemment les résultats selon les proportions relatives au sein du Collège Électoral (illustration 2) ou bien selon la population de chacun des États (illustration 3), et a fortiori pour la présidentielle de 2020.

Illustration 1 - Résultats de l'élection présidentielle 2020 par États

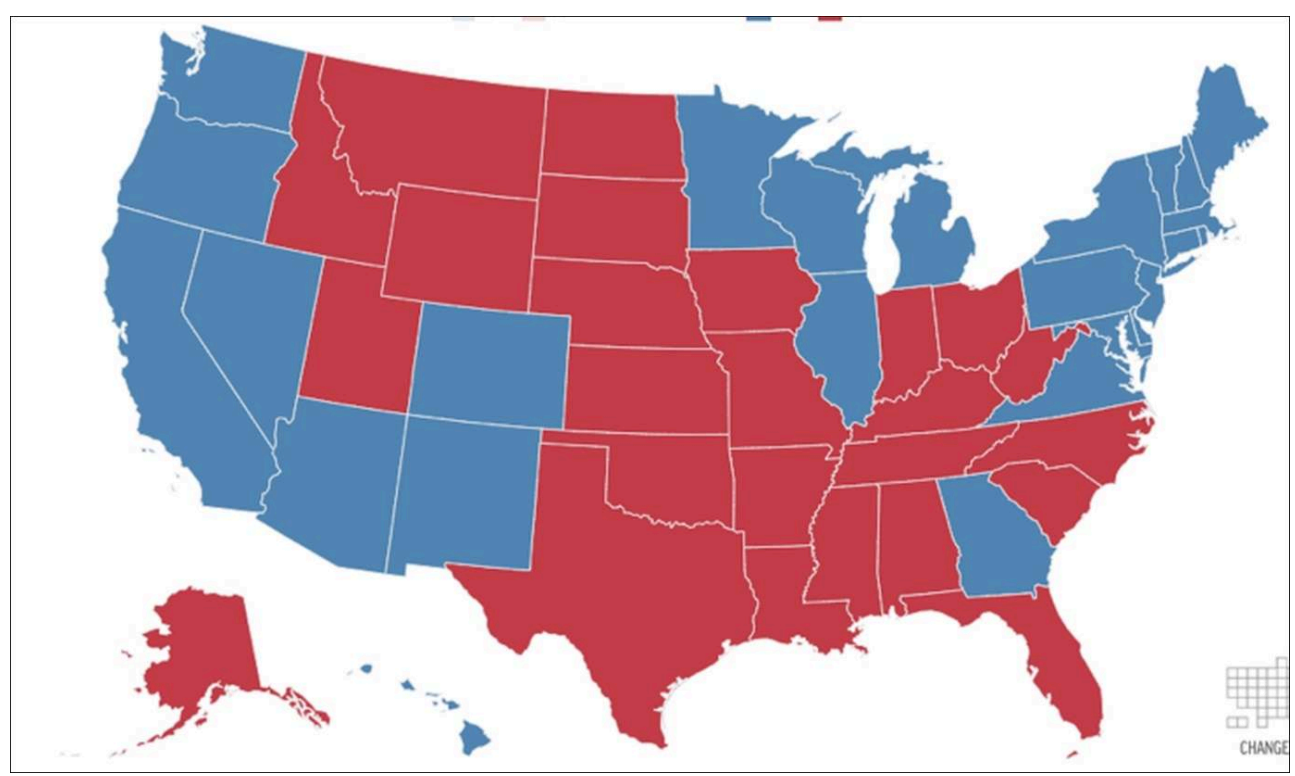

Bleu : États remportés par les Démocrates; Rouge : États remportés par les Républicains.

Source : CSpan, https://www.c-span.org/election/ (consulté en décembre 2020). 
Illustration 2 - Résultats de l'élection présidentielle 2020 (nombre de voix au sein du collège électoral, par État)

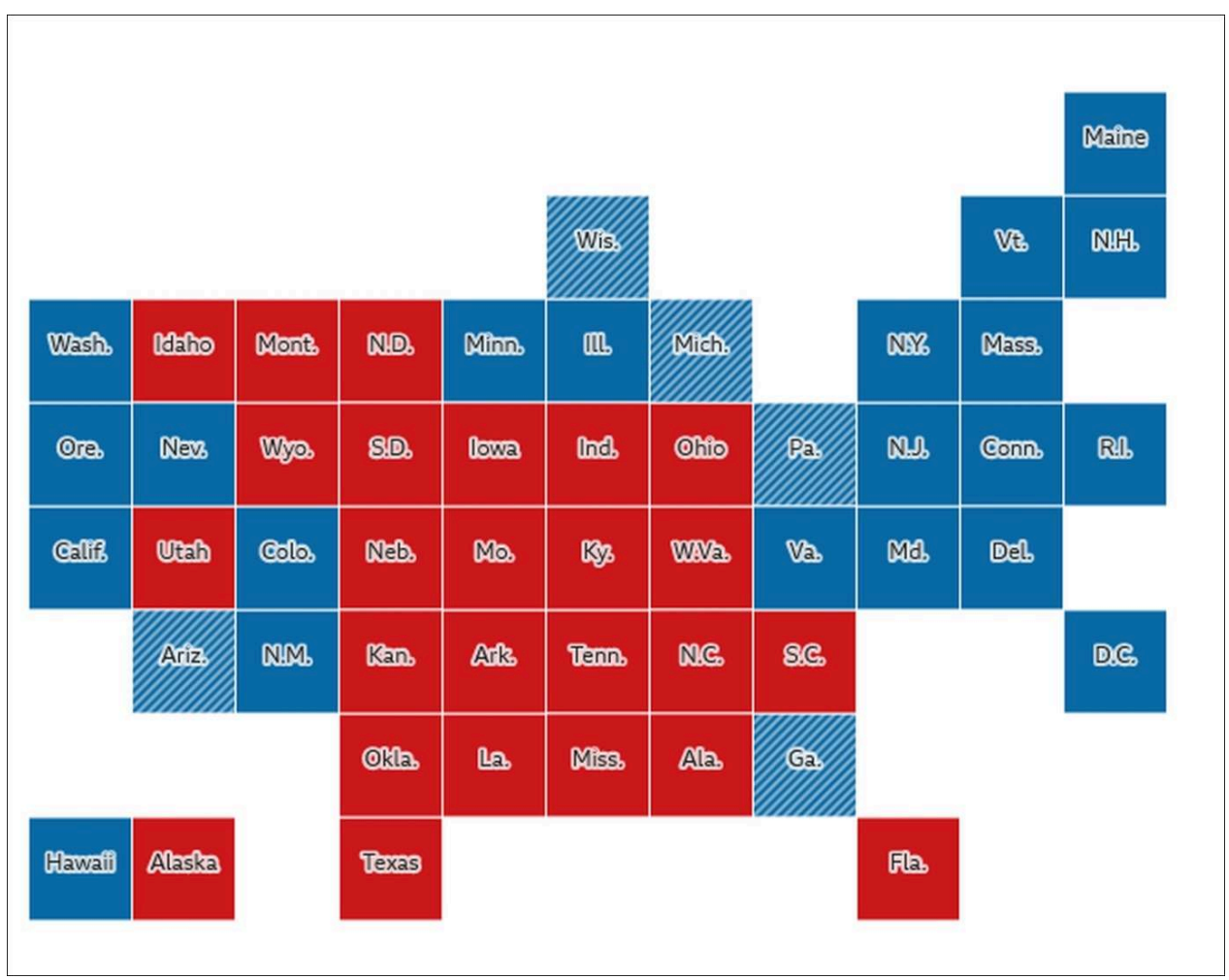

Bleu : États remportés par les Démocrates; rouge : États remportés par les Républicains ; hachures bleues : États républicains qui basculent côté démocrate.

Source : https://www.bbc.com/news/election-us-2020-54783016

Illustration 3 - Cartogramme du résultat de l'élection présidentielle 2020 (vote selon la population respective de chacun des États)

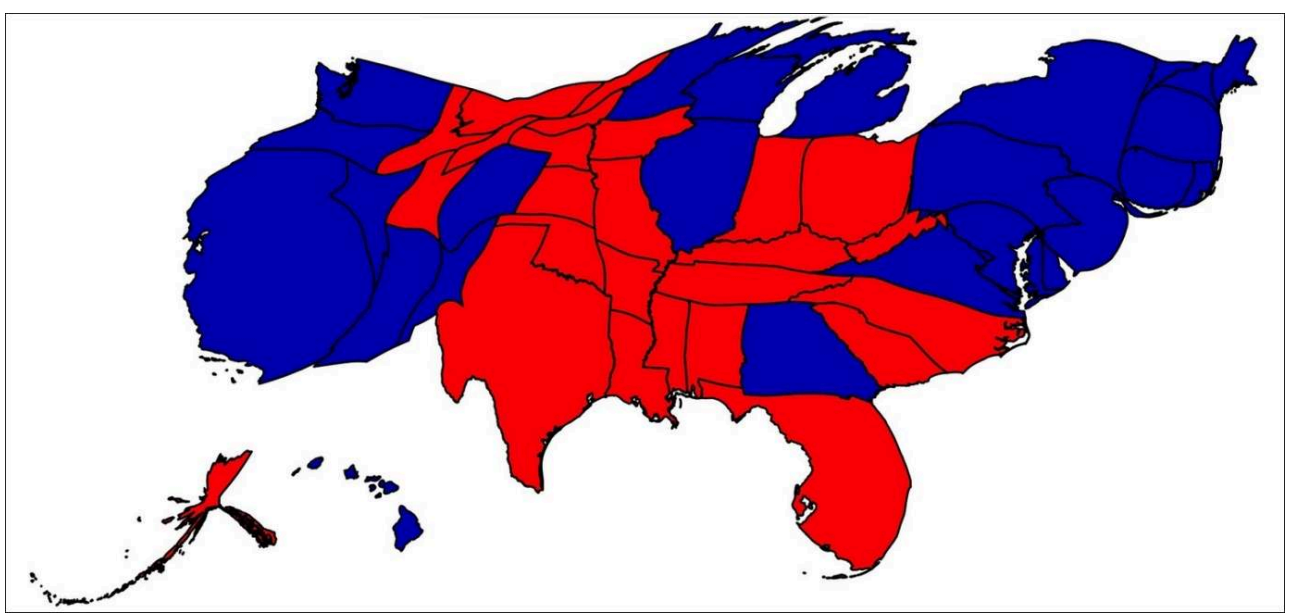

Bleu : États remportés par les Démocrates; Rouge : États remportés par les Républicains.

Source : https://medium.com/swlh/how-the-people-really-voted-bfdd8aa872f2 
Illustration 4 - Degrés de possibilité d'utiliser le vote par correspondance

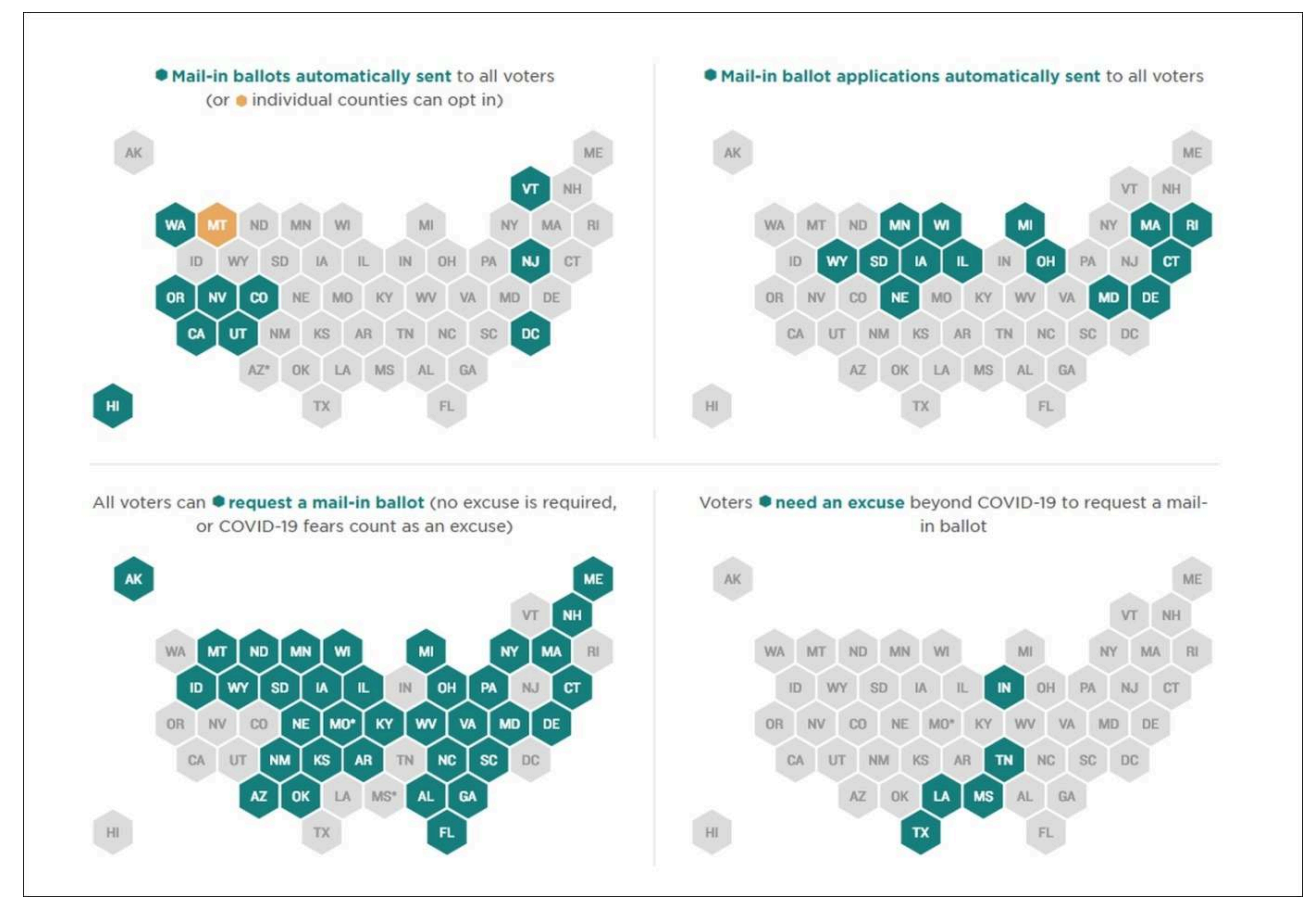

Carte haut gauche : hexagones/États verts : les bulletins de vote par correspondance sont postés directement aux électeurs ; État/hexagone orange : les comtés peuvent choisir cette option. Carte haut droite : hexagones/États verts : des formulaires de demande de vote par correspondance sont automatiquement postés à tous les électeurs.

Carte bas droite : États/hexagones verts : un motif autre que le Covid-19 doit être fourni pour faire une demande de vote par correspondance.

Carte $b$ gauche : hexagones/États verts : tous les électeurs peuvent demander à voter par correspondance en invoquant le Covid-19 ou une autre raison acceptée.

Source : https://www.npr.org/2020/09/14/909338758/map-mail-in-voting-rules-by-state? $\mathrm{t}=1609334704712$

5 L'augmentation du taux de participation peut être attribuée à une nette recrudescence du vote par correspondance pour des raisons conjoncturelles. En effet, le recours à cette forme de vote a été encouragée dans la plupart des États aux prises avec une situation sanitaire inquiétante en pleine pandémie de Covid-19. Ainsi, la majorité des États a mis en place des mesures très concrètes pour faciliter le vote à distance (illustration 4). Plusieurs États ont systématiquement posté des formulaires de demande de vote par correspondance à tous les électeurs inscrits, dans le nord-est et le nord du Midwest (Wisconsin, Michigan par exemple). D'autres États ont, eux, posté directement les bulletins de vote aux électeurs inscrits, principalement les États de l'Ouest (Californie, Colorado, Utah, Nevada, Oregon). La quasi-totalité des États a, de surcroît, autorisé les électeurs à demander à voter par correspondance sans avoir à donner de raison autre que celle liée au Covid-19, sauf cinq États: Texas, Louisiane, Mississippi, Tennessee, au sud, et l'Indiana (état du vice-président Pence). Ces cinq États sont tous républicains. Cette facilité de recours au vote par correspondance, pourtant justifiée par l'incertitude sanitaire, a été violemment dénoncée par Donald Trump dès le printemps 2020 puisqu'il y voyait une possibilité de fraude: bulletins inventés, bourrage d'urnes, électeurs fictifs ${ }^{3}$. Il déclarait, en effet, au cours d'une interview qu'il accordait à l'émission Fox \& Friends, que les sommes prévues dans le CARES Act $^{4}$ en mars 2020 par le Congrès pour soutenir l'effort logistique et 
organisationnel nécessaire à la mise en place d'un vote par correspondance dans la plupart des États montrait que "Ce qu'ils [les Démocrates, ndlr] avaient mis là-dedans, c'était dingue. Il y avait des trucs - des niveaux de participation au vote qui, si on les acceptait, plus jamais un Républicain ne serait élu dans ce pays $»^{5}$. Donald Trump s'engage dès lors dans de véritables joutes verbales qui visent à installer un doute sur la régularité du vote et qui durera jusqu'à l'élection, et au-delà.

Pour donner crédit à ses invectives il s'appuie sur ce que la carte «totalisante » des résultats du Collège Électoral (illustration 1) ne peut pas montrer. C'est, en fait, une très grande disparité que l'on observe au sein de chaque état, et particulièrement dans les États pivot. En effet, l'illustration 1, officielle, ne permet pas de comprendre les tensions, très fortes en 2020, et les prises de position, parfois radicales, qui s'ancrent dans des situations géographiques, donc économiques et sociales, extrêmement contrastées. Par ailleurs, comme nous l'avons vu précédemment, elle ne permet pas non plus de rendre compte de la taille de la population. Et, en cela, masque ou amoindrit visuellement le résultat du vote populaire. En 2020, il y a adéquation entre le vainqueur du scrutin populaire et du Collège Électoral. En 2016, les deux résultats étaient disjoints. Donald Trump avait remporté une majorité au sein du Collège Électoral mais était arrivé second, derrière Hillary Clinton, pour le vote populaire ${ }^{6}$.

7 Tous ces angles morts de la carte officielle des résultats alimentent les souhaits de réforme du Collège Électoral. De nombreux juristes tels qu'Edward Foley, ou historiens tels que Jack Rakove, appellent à une véritable refonte du système électoral qui, au plan national, ne paraît plus représentatif. Rakove souligne que :

Les États ne sont que les divisions géographiques arbitraires qui déterminent la façon dont les préférences individuelles sont distribuées dans le pays. Le but d'une réforme électorale optimale devrait permettre de traduire, au niveau national, l'expression de ces préférences additionnées grâce à un vote populaire dont la circonscription sera l'État fédéral tout entier. ${ }^{7}$

8 Parallèlement Alexander Keyssar (2020) explique lui aussi, dans son dernier ouvrage, les raisons qui ont mené le Congrès à essayer de réformer le Collège Électoral et pourquoi ces tentatives, à ce jour, ont toutes échoué. Il propose une alternative à l'action législative, vouée à l'échec, qu'il nomme National Popular Vote Interstate Compact.

Effectivement, scrutés plus finement, à l'échelon de l'état et des comtés, les résultats prennent un autre sens. Tout particulièrement dans les États pivots où seule une cartographie à une autre échelle permet de comprendre les enjeux de l'élection, et du basculement de l'État dans un camp ou l'autre.

\section{Effets d'échelle : l'État et le comté}

On constate que, pour une écrasante majorité d'États (une quarantaine sur les cinquante), il n'y a pas de doute quant au résultat de l'élection ${ }^{8}$. Les candidats n'y font d'ailleurs presque pas campagne, ne s'y rendent que très rarement, car ils savent que le résultat leur est acquis. L'élection fédérale se joue donc dans quelques États dits pivots. L'étude de cette caractéristique est l'un des apports fondamentaux de la théorie du réalignement électoral (electoral realignment) formulée à la suite d'une élection déterminante (critical election). À partir des années 1950, plusieurs politistes, dans le sillage des travaux de V.O. Key, théorisent le réalignement électoral. Ils montrent qu'un petit nombre d'élections réalignent les tendances et les appartenances pour les 
Démocrates ou les Républicains. Ces élections (principalement au tournant des années 1850, 1890, 1930 et 1970) sont caractérisées par un taux de participation élevé, une sensibilisation plus radicale aux enjeux de campagne et des résultats qui montrent un clivage net par rapport à l'élection précédente ${ }^{9}$. Si cette théorie, dans son acception première, fait aujourd'hui débat, elle permet néanmoins de s'interroger sur les causes et les effets de la polarisation croissante qui caractérise depuis plusieurs années la scène politique américaine. Cette polarisation est saisissante si l'on étudie les cartes détaillées des résultats dans les États pivot, où les marges de victoire tant pour Biden que pour Trump sont très, voire extrêmement faibles. Quelques milliers de voix, des écarts qui oscillent entre $0,2 \%$ et moins de $2 \%$ séparent les deux candidats.

Pennsylvanie (illustration 5a): Biden 50,01\% - Trump 48,84\% - Jorgensen (Parti libertarien) $1,15 \%$;

Michigan (illustration 5b) : Biden 50,63\% - Trump 47,85\%;

Wisconsin (illustration 6a) : Biden 49,57\% - Trump 48,94\%;

Géorgie (illustration 6b): - Biden 49,5\% - Trump 49,26\% - Jorgensen (Parti libertarien) $1,25 \%$

L'écart entre Biden et Trump est de 11779 voix $^{10}$;

Floride (illustration 7a) : - Biden 47,87\% - Trump 51,22\%;

Arizona (illustration 7b) : Biden 49,39\% - Trump 49,09\% - Jorgensen (Parti libertarien) $1,52 \%$ (10 457 voix d'écart entre les deux candidats sur un total de suffrages exprimés de 3334 829);

Nevada (illustration 11) : Biden 50,06\% - Trump 47,67\% - Jorgensen (Parti libertarien) 1,05\% - Don Blankenship (Parti de la Constitution) 0,22\% ${ }^{11}$.

Illustration 5 a et b - Résultats de l'élection présidentielle 2020 en Pennsylvanie (gauche) et dans le Michigan (droite)
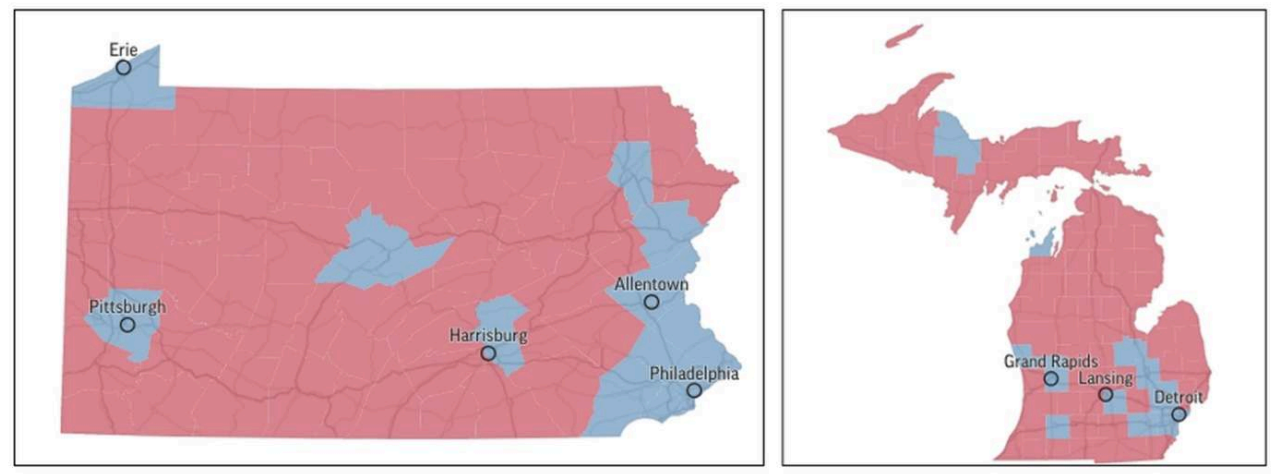

Bleu : comtés remportés par les Démocrates ; Rouge : comtés remportés par les Républicains Source : https://www.c-span.org/election/ (consulté en décembre 2020) 
Illustration 6 a et b - Résultats de l'élection présidentielle 2020 dans le Wisconsin (gauche) et en Géorgie (droite)
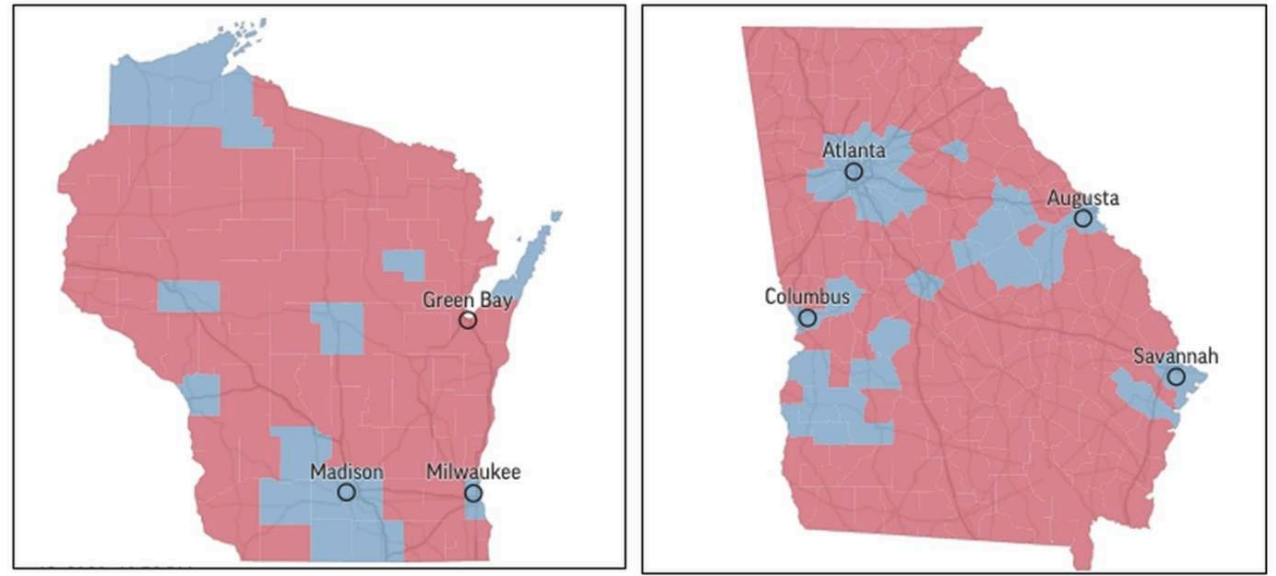

Bleu : comtés remportés par les Démocrates ; Rouge : comtés remportés par les Républicains Source : https://www.c-span.org/election/ (consulté en décembre 2020)

Illustration 7 a et b - Résultats de l'élection présidentielle 2020 en Floride (gauche) et dans l'Arizona (droite)
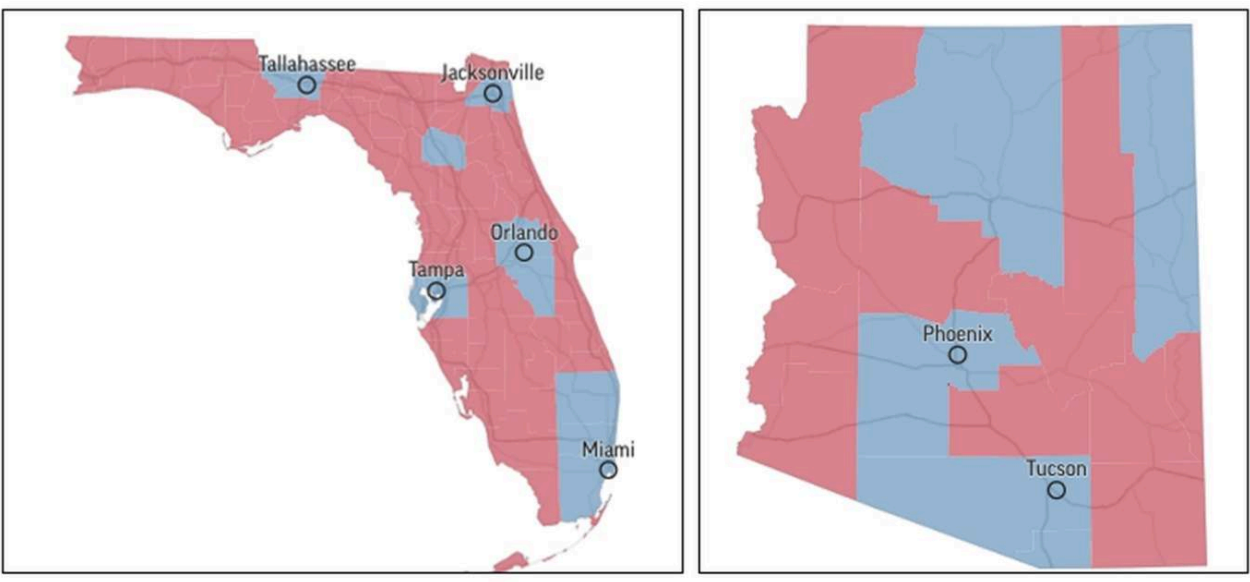

Bleu : comtés remportés par les Démocrates; Rouge : comtés remportés par les Républicains Source : https://www.c-span.org/election/ (consulté en décembre 2020) 


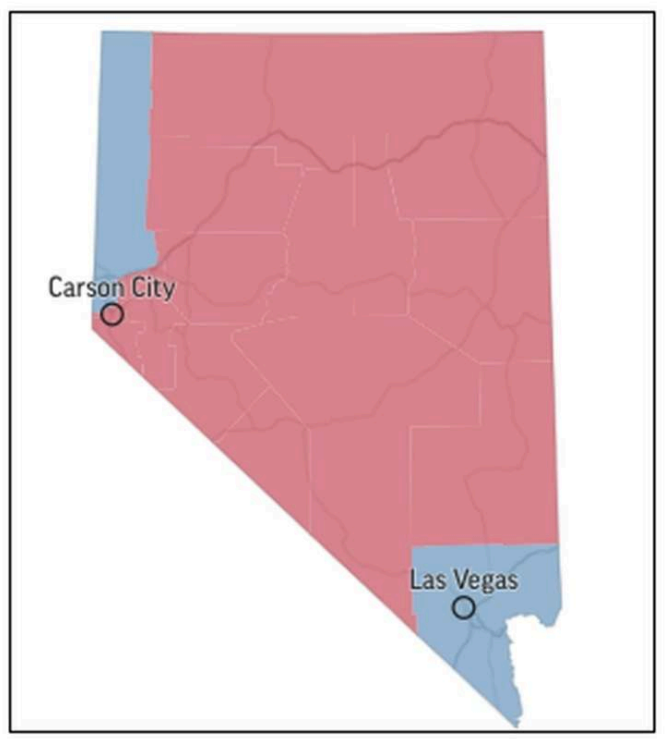

Bleu : comtés remportés par les Démocrates; Rouge : comtés remportés par les Républicains Source : https://www.c-span.org/election/ (consulté en décembre 2020) chacun de ces États. Ajoutée à des écarts de voix très faibles, on comprend qu'ils aient été au centre du suspense qui s'est poursuivi pendant plusieurs jours après le 3 novembre. En effet, il a fallu attendre le 7 novembre pour avoir une quasi-certitude sur l'issue du scrutin ${ }^{12}$. Ce que les chiffres ne montrent pas, en revanche, et que seule une carte détaillée de chacun des États peut laisser voir, c'est la forte polarisation au sein même des États. D'un comté à l'autre, les résultats sont très tranchés. Comme on peut le constater, les zones urbaines votent très massivement démocrate et les zones plus rurales votent Républicain, les États pivot ne font pas exception. Sur les 3006 comtés qui composent les États-Unis ${ }^{13}, 247$ comtés ont changé de parti dominant (soit $8,2 \%$ des comtés) entre 2012 et 2016. Parmi ceux-ci, 217 ont soutenu Donald Trump en 2016 après avoir soutenu Barack Obama auparavant (2012) ${ }^{14}$. En 2020, le nombre de comtés " pivot » est tombé à $75: 60$ d'entre eux sont passés de Trump (rouge) à Biden (bleu) et 15 comtés de Biden (bleu) à Trump (rouge), dont 8 dans l'État du Texas, comtés constitués en forte proportion d'électeurs Latino de la classe ouvrière ${ }^{15}$. Par exemple, le comté de Zapata à l'extrême sud du Texas n'avait pas voté Républicain depuis Warren Harding dans les années $1920^{16}$. Trump a effectivement gagné des voix parmi l'électorat latino, et c'est aussi un enseignement de l'élection de 2020. Cet enseignement devra être apprécié à sa juste mesure étant donné les tendances démographiques (croissance supérieure à la moyenne nationale) de la population latino aux États-Unis. On peut voir, d'ailleurs, dans les nominations de Biden pour son gouvernement, le signe très clair d'une volonté d'inclure plus de latinos $^{17}$.

Si l'on prend le cas de l'Arizona, un comté représente, à lui seul, $60 \%$ des votes de l'État. Il s'agit du comté de Maricopa, où l'on trouve la grande ville de l'État: Phoenix. Ce comté, principalement urbain, a été remporté par Biden. Il est le premier candidat démocrate depuis Harry Truman en 1948 à remporter ce comté. Cela lui a permis de 
remporter cet État (illustration 7b). Dans le Michigan (illustration 5b), c'est le comté de Kent où l'on trouve la ville de Grand Rapids qui a permis de faire la différence dans cet État qui avait été remporté par Trump en 2016. Dans le Wisconsin (illustration 6a), deux comtés, remportés par Biden, lui ont valu de gagner cet État. Le comté de Milwaukee, où se trouve la ville du même nom, et le comté de Dane, qui abrite l'Université du Wisconsin à Madison, ont voté plus fortement démocrate qu'en $2016^{18}$. On constate donc qu'au sein des États pivots, ce sont quelques comtés seulement qui basculent dans un camp ou l'autre, qui décident du résultat, portant ainsi la focale électorale sur un territoire très restreint. Si les Démocrates sont parvenus à faire basculer certains comtés clé en leur faveur et à remporter certains États grâce au vote urbain, il n'en reste pas moins que le paysage électoral semble se figer autour de constantes désormais bien arrêtées.

Ce sont les conséquences géographiques de la situation socio-économique d'un électorat désabusé que Trump a bien intégrées (voir par exemple, Achen \& Bartels, 2017). Sous les effets croisés de la mondialisation, des chocs financiers et de l'automatisation des tâches, toute une frange de la population américaine a été touchée par, et/ou vit dans, la crainte d'une déchéance économique et sociale. Face à cette situation, les discours positivants des Démocrates ne suffisent plus et c'est dans cette brèche que s'est engouffré Donald Trump (Voir par exemple, Barker, 2012). Lorsqu'il parle de carnage ou à l'inverse avance son slogan de "MAGA - Make America Great Again ", c'est bien ce sentiment de déclin qu'il veut convertir en capital politique. Anne Case et Angus Deaton analysent ce qu'ils appellent les "morts dues au désespoir " (deaths of despair). Cette mortalité en croissance est mue par un désespoir qui conduit à une surconsommation d'opioïdes. Avant la pandémie, le Council on Foreign Relations déclarait que « la crise a atteint une telle ampleur qu'elle est une menace pour l'économie et pour la sécurité nationale $»^{19}$. Le président Trump créait, en 2017, une commission pour étudier et combattre les effets dévastateurs de la crise des opiacés ${ }^{20}$. La répartition géographique de leur consommation est éloquente si l'on regarde la carte réalisée par l'agence gouvernementale Center for Disease Control (illustration 9). Cette consommation est forte dans des États du Sud, de l'Ouest et du Midwest, ayant un tissu urbain moins développé, et qui affichent tous une majorité républicaine. Il faut ajouter à cette situation dramatique la part du coût des dépenses de santé dans le budget des Américains. Elle est passée de $5 \%$ en 1960 à près de $18 \%$ du revenu national aujourd'hui ${ }^{21}$ (avant la mesure des effets de la pandémie). Certains candidats démocrates (dont Bernie Sanders, le sénateur du Vermont) soutiennent l'idée d'une couverture médicale universelle (Medicare for all) mais les moyens de financer ce programme restent assez flous et cette initiative ne permet pas aux Démocrates d'emporter une adhésion massive auprès de la population, et particulièrement des électeurs les plus touchés qui, dans bien des cas, votent Républicain.

Comme en attestent les cartes, la forte polarisation entre Démocrates et Républicains dans la majorité des États a creusé de profonds sillons sur le territoire américain. Cette tendance est aussi à l'œuvre pour les élections au Congrès, et plus précisément à la Chambre des représentants. 


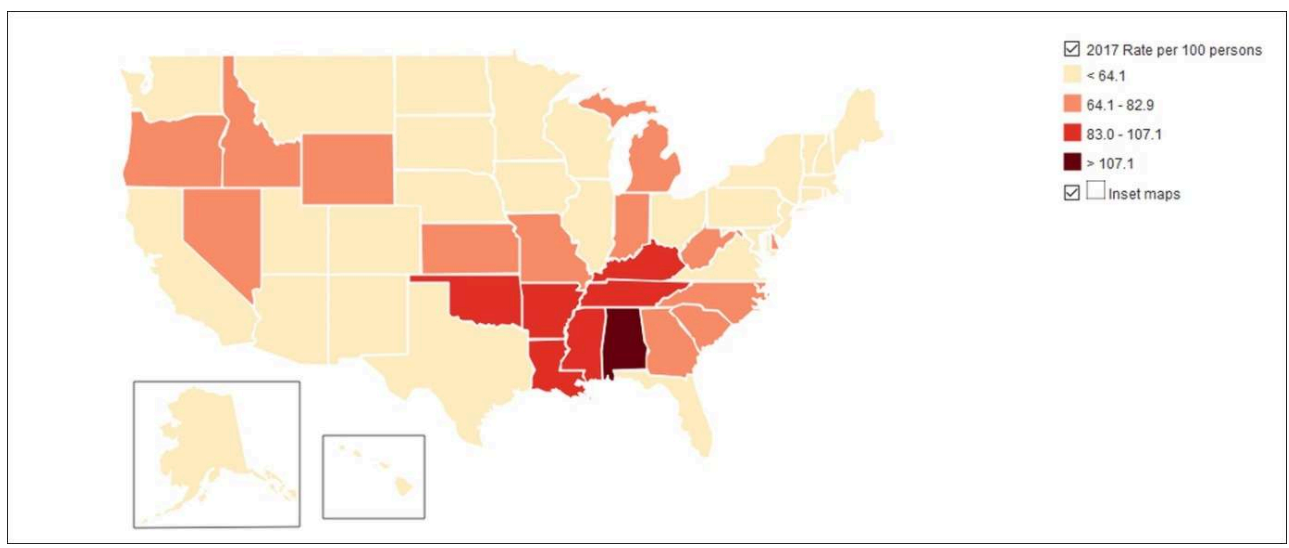

Pourcentage d'ordonnances prescrivant des opiacés (par personne et par an (en 2017).

Source : Center for Disease Control, https://www.cdc.gov/drugoverdose/maps/rxstate2017.html

\section{Élections au Congrès et cartes des Congressional Districts}

Si les media américains et internationaux étaient rivés aux résultats de l'élection présidentielle du 3 novembre 2020, les électeurs, eux, se prononçaient, le même jour, et sur le même bulletin de vote, pour plusieurs autres fonctions électives. Ils devaient voter pour élire leur député à la Chambre des représentants au Congrès à Washington, et dans un tiers des États, leur sénateur ${ }^{22}$. Les électeurs se prononçaient aussi pour toute une série d'autres fonctions locales.

Les résultats des élections à la Chambre ont été décevants pour les Démocrates. Certes, ils conservent la majorité, mais elle s'est effritée. Battus dans plusieurs circonscriptions, les 222 députés démocrates dépassent de peu le chiffre clé de 218 sièges nécessaires à la majorité. Les Républicains, quant à eux, remportent dix circonscriptions pour atteindre le chiffre de 211 députés. Comme le montre la carte des résultats (illustration 10), la poussée républicaine se confirme dans la granularité23 des circonscriptions (Congressional Districts) au Nouveau-Mexique, dans le Minnesota, dans l'Iowa, ou encore en Floride. La carte des circonscriptions électorales est encore plus rouge que la carte des résultats de la présidentielle. Pourtant, dans les deux cas, les Démocrates l'emportent. 
Illustration 10 - Résultats des élections législatives (Chambre des représentants à Washington), 3 novembre 2020

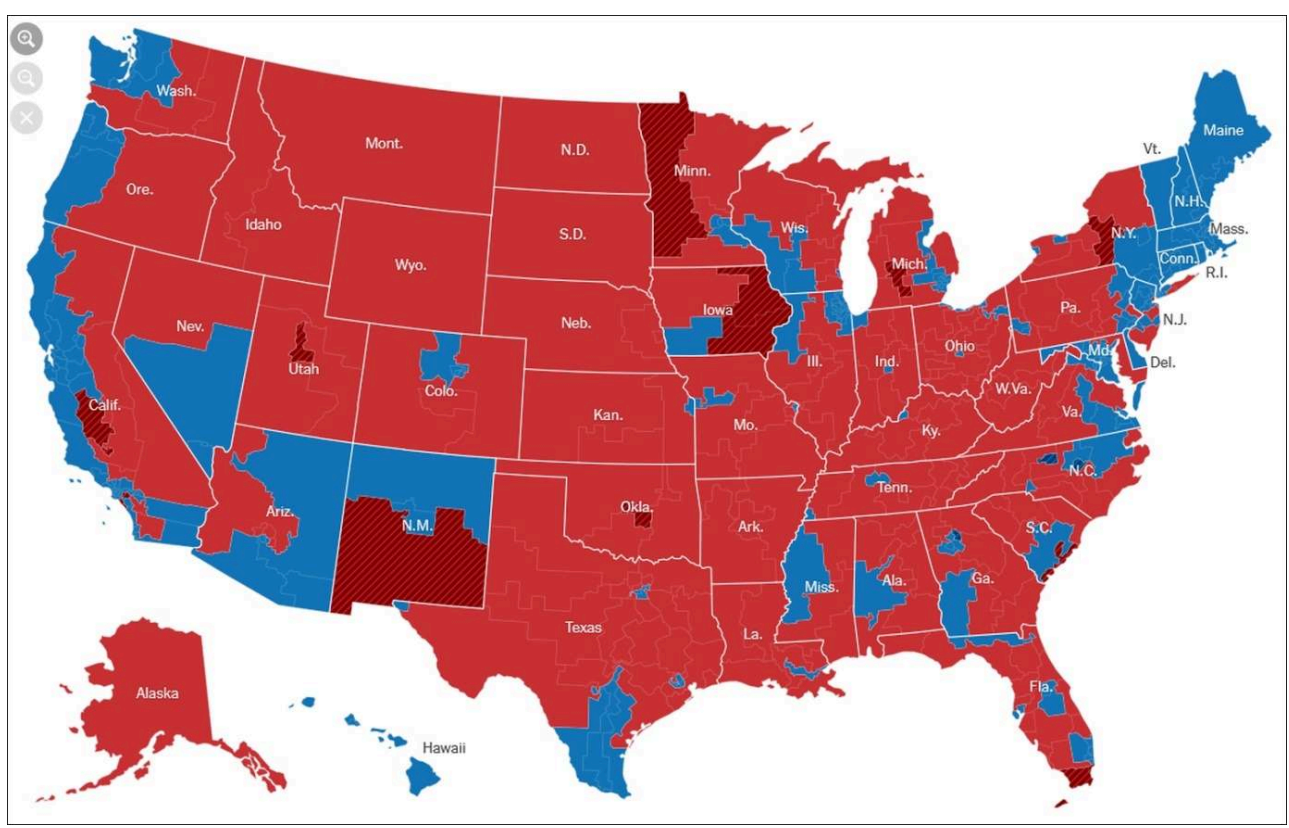

Bleu : circonscriptions électorales remportées par les Démocrates ;

Rouge : circonscriptions électorales remportées par les Républicains.

Hachures rouges : circonscriptions qui basculent côté républicain

Hachures bleues : circonscriptions qui basculent côté démocrate.

Hachures grises : les résultats très serrés de la 2ème circonscription de l'lowa ont donné lieu à une action en justice. La candidate démocrate s'est désistée le 31 mars 2021.

Source : https://www.nytimes.com/interactive/2020/11/03/us/elections/results-house.html

Selon Alan Schweber (2016, p.381), «Ce que le cartographe décide d'inclure [sur une carte] n'est rendu visible que grâce à l'exclusion de tous les autres sujets possibles ${ }^{24}$. Le cartographe qui suit le texte constitutionnel élude, sur la carte nationale, ce que seules les cartes des États fédérés, et encore plus des circonscriptions électorales au sein de ces mêmes États, peuvent révéler. Ainsi, les Démocrates ont perdu des sièges de députés dans des circonscriptions à tendance conservatrice en Caroline du Sud, dans l'Iowa et le Nouveau-Mexique, mais aussi en Californie et en Floride. Cela donne une majorité démocrate à la Chambre qui est la plus faible enregistrée au cours des 18 dernières années ${ }^{25}$. Or, au-delà des chiffres et de la taille de la délégation démocrate ou républicaine au Congrès à Washington, c'est encore dans chacun des États qu'il faut chercher les raisons de ces résultats, de ces glissements dans les cartes.

\section{Cartes des Congressional Districts (CDs)}

Le nombre de députés de chacun des États est proportionnel à la population de l'État ${ }^{26}$. Chaque député fédéral siégeant à la Chambre des représentants à Washington est élu dans sa circonscription (Congressional District) au sein de son État d'appartenance (home state). Et c'est le recensement décennal de la population américaine tel qu'il est prévu par la Constitution ${ }^{27}$ qui permet de faire les ajustements nécessaires lorsque certains États gagnent en population alors que d'autres se dépeuplent. Il s'instaure donc un mouvement dynamique de gain, ou de perte, de députés pour certains États, dont le recensement rend compte tous les dix ans. L'année 2020 est, en cela aussi, une année importante car les résultats du recensement qui vient d'avoir lieu vont mettre en 
évidence les États qui auront gagné des députés et ceux qui en auront perdu. Cela signifie également que plusieurs États vont être obligés de redécouper leurs Congressional Districts, pour en augmenter ou en diminuer le nombre.

La question épineuse des découpages électoraux et, donc, la transformation parfois profonde des cartes des CDs, traverse toute l'histoire américaine. Elle a souvent été le creuset territorial d'inégalités et de discriminations dans la représentation des électeurs. Aujourd'hui encore, le débat fait rage entre Démocrates et Républicains, dans les États fédérés, sur les modes de découpage et de redécoupage des circonscriptions. Les cartes des circonscriptions sont autant de tests pratiques de la qualité, et de la réalité, de la représentation électorale.

\section{Cartes et discrimination raciale}

La carte des CDs a été, et reste encore aujourd'hui, le théâtre de distorsions importantes visant à limiter, ou nier, l'expression et le vote (illustration 11$)^{28}$. Ainsi, dans de nombreux États, l'absence de découpages jusqu'au milieu des années 1960 dans certains cas a réduit les cartes au silence. Immobilisées et figées, les cartes des circonscriptions électorales avaient perdu tout lien avec la réalité de la population qu'elles étaient censées représenter. La ségrégation et la séparation entre « whites » et « coloured $»^{29}$ Jim Crow laws - et la volonté de priver la population noire de l'accès au vote étaient signifiées sur les cartes. Une frange de la population de certains États était invisibilisée sur les cartes. À la faveur du mouvement pour la défense des droits civiques, et face à ce déséquilibre, se met en marche une offensive cartographique qui permet de rétablir, peu à peu, l'égalité des votes et de la représentation. Les cartes des circonscriptions électorales qui avaient privé certains électeurs de leur voix pendant des décennies sont, une à une, redécoupées et remises en mouvement. Plusieurs actions en justice sont intentées, en particulier dans des États du Sud, pour dénoncer des cartes de circonscriptions qui aboutissent à une représentation inégalitaire et discriminante. C'est grâce à cette volonté politique de réécrire les cartes officielles et de modifier les contours des circonscriptions que la représentation retrouve peu à peu son territoire. Les trois recensements de 1970, 1980 et 1990 permirent de dessiner des cartes rétablissant tout ou partie de la pluralité représentative de la population noire. 
Illustration 11 - Carte des circonscriptions électorales (Congressional Districts), $117^{\text {ème }}$ Congrès (2021-23)

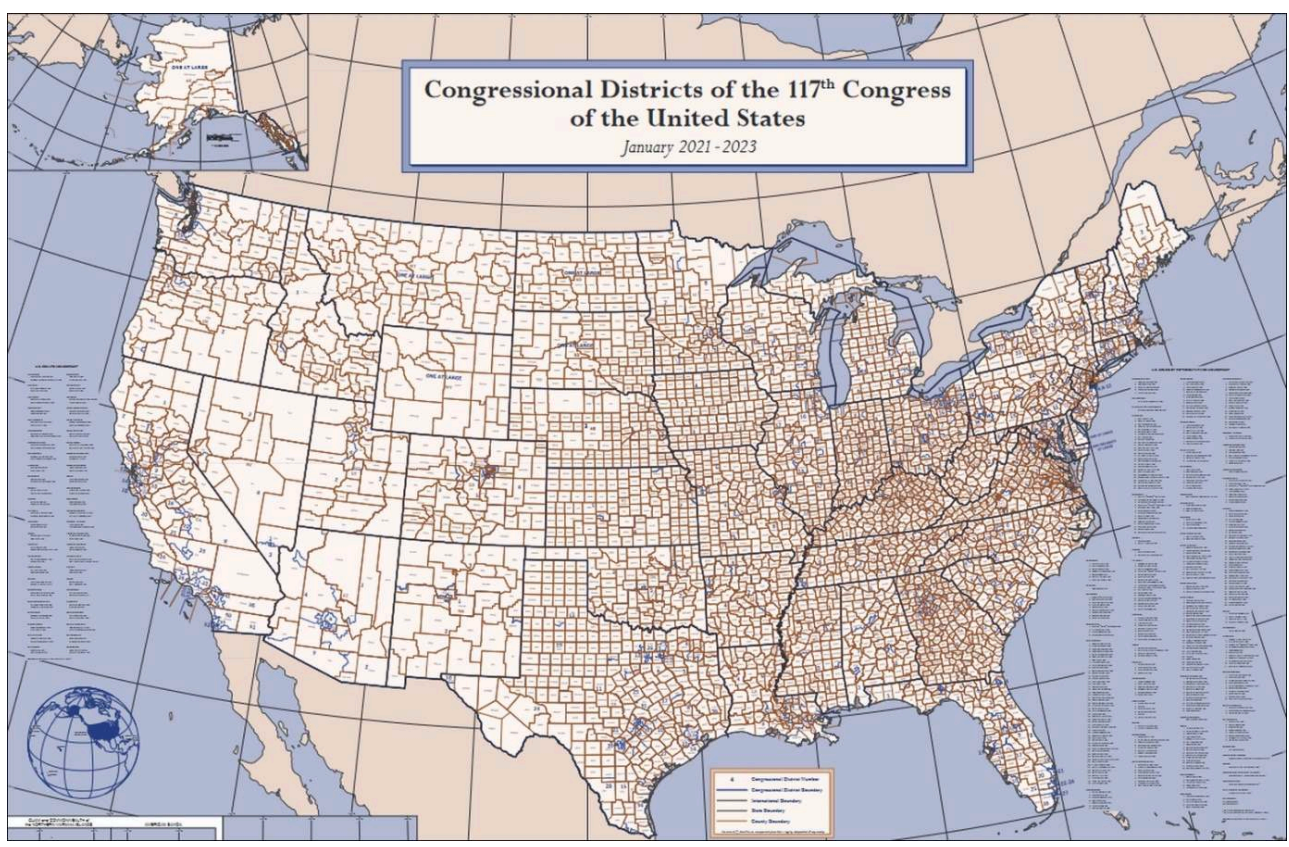

Source: Bureau of the Census, https://www2.census.gov/geo/maps/cong_dist/uswall/cd117/ CD117_US_WallMap.pdf

\section{Cartes et découpages partisans, une autre forme de discrimination}

Cependant, à partir des années 2000, c'est une autre source de discrimination qui se fait jour au travers des cartes électorales. En effet, alors qu'on assiste à une érosion démographique dans les États du Nord et de l'Est, les États du Sud et de l'Ouest voient leur population augmenter ${ }^{30}$. Cela conduit à une perte de députés dans les régions du Nord Est, qui doivent donc supprimer des circonscriptions électorales. On constate parallèlement une augmentation du nombre de députés dans certains États du Sud et de l'Ouest, où il faut créer des circonscriptions nouvelles.

Les manipulations cartographiques, dès lors, ne s'ancrent plus, ou moins directement, dans un terreau de discrimination raciale, mais dans une discrimination partisane. On dénonce, en effet, des découpages qui conduisent à une discrimination contre les Démocrates, ou contre les Républicains, suivant les États. La recrudescence des redécoupages, qui va d'ailleurs se poursuivre à la suite du recensement de 2020, s'accompagne de doutes pesants entourant le soupçon de charcutage électoral à motifs partisans. Les cartes redeviennent un champ de bataille, tant pour les Républicains que pour les Démocrates. Si la bataille se joue dans les assemblées des États fédérés, en charge des redécoupages, ses effets se produisent à Washington, comme l'ont montré les résultats des élections de l'automne 2020 (illustration 10).

Puisque c'est le contrôle de la majorité à la Chambre des représentants qui est en jeu, le redécoupage électoral est devenu une affaire de haute précision. Le découpage «high tech », alimenté par des données très détaillées, est plus minutieux encore, mais aussi, paradoxalement, plus manipulable. Comme le soulignent Turner et LaMacchia (1999), depuis la mise en place de redécoupages assistés par ordinateur et de logiciels qui permettent de croiser des données démographiques et des données cartographiques, la 
technicité du processus pourrait laisser croire que la neutralité s'est installée. Or, il n'en est rien. La puissance de cette technicité nouvelle ne saurait masquer les intentions, issues de tout bord, qui peuvent conduire à des gerrymanderings ${ }^{31}$. La " géoépistémologie " (Rankin, 2016) des cartes redécoupées se poursuit donc en essayant de parfaire les outils cartographiques utilisés. En effet, pour dissiper les suspicions de charcutages, des solutions reposant sur l'utilisation de logiciels sophistiqués sont proposées dans plusieurs États. Ainsi, TIGER ${ }^{32}$ (Topologically Integrated Geographic Encoding and Referencing), est devenu l'outil de travail quotidien du bureau du recensement (Census Bureau). TIGER donne des informations extrêmement fines concernant la topographie du territoire mais aussi les circonscriptions électorales rapportées à chacune des sessions du Congrès à Washington ${ }^{33}$. Le recours à un SIG (Système d'Information Géographique) n'est pas nouveau, mais, en revanche, l'intensité avec laquelle les SIG sont désormais utilisés par les partis et législatures d'État devant procéder à des redécoupages est un phénomène nouveau. Plusieurs outils concurrents sont ainsi disponibles. On citera, par exemple, ESRI, Azavea, Arcbridge, ou encore Zillioninfo ${ }^{34}$.

Les deux partis se sont donc emparés de ces interfaces techniques et deux organisations partisanes sont particulièrement actives dans les redécoupages électoraux. Côté démocrate, le National Democratic Redistricting Committee ${ }^{35}$ placé sous la houlette d'Eric Holder $^{36}$ et, côté républicain, le REDistricting MAjority Project (REDMAP) ${ }^{37}$ dont le nom traduit la volonté affichée de transformer le plus de circonscriptions possibles en territoires « rouges» donc républicains. Barack Obama rappelait lors de son dernier discours sur l'état de l'Union, en 2016, qu'il était nécessaire de « changer le système (...) et mettre fin à la pratique qui consiste à dessiner nos circonscriptions de manière à ce que ce soit les hommes politiques qui choisissent leurs électeurs et non l'inverse. Il faut s'en remettre, pour cela, à un groupe bipartisan ${ }^{38}$. Plus que jamais, et malgré le regain d'activité cartographique depuis près de 60 ans dans ce domaine, les efforts, et les attaques, se poursuivent pour s'emparer du tracé des lignes sur les cartes.

\section{Redécoupages après le recensement de 2020}

En 2019 a été lancée une campagne nationale visant à mettre en lumière, et à prévenir, les charcutages électoraux potentiels qui pourraient survenir après la publication des résultats du recensement décennal de $2020^{39}$. Cette campagne porte le nom de All On The Line et elle veut attirer l'attention de la population américaine et de l'électorat dans son ensemble sur les circonscriptions sensibles qui pourraient donner lieu à des manœuvres cartographiques contestables. Dix États sont concernés: la Floride, la Géorgie, la Caroline du Nord, la Pennsylvanie, l'Ohio, le Michigan, le Wisconsin, le Colorado, l'Arizona et le Texas. L'objectif affiché de AOTL ${ }^{40}$ est clair :

«Une personne, une voix [...]. Il y a une barrière structurelle fondamentale qui empêche le progrès: des cartes électorales biaisées, tracées avec une précision chirurgicale par des politiciens afin de préserver leur pouvoir politique et de réduire au silence la volonté du peuple ».

Dans les États pivot, la fébrilité et l'empressement sont grands, dans un camp comme dans l'autre, pour surveiller les redécoupages électoraux. Or, puisque dans une écrasante majorité d'États, c'est la législature ${ }^{41}$ d'état qui est en charge du redécoupage électoral, c'est donc le parti majoritaire, aux commandes, qui préside au nouveau découpage. C'est pourquoi, afin de découpler pouvoir, d'une part, et responsabilité du 
découpage, d'autre part, plusieurs États ont mis en place des systèmes alternatifs tels que des redistricting commissions (commissions chargées du redécoupage) ${ }^{42}$ afin d'adopter une forme de neutralité dans les opérations cartographiques ${ }^{43}$. Les deux obstacles principaux qui vont accompagner chacun des redécoupages à venir sont, premièrement, les poursuites légales visant à invalider les cartes proposées par l'un ou l'autre des partis au pouvoir, et, deuxièmement, la nécessité de lever des fonds pour rémunérer les équipes techniques et juridiques qui vont soumettre des propositions de redécoupage aux différentes assemblées d'état ou commissions chargées du redécoupage. Sur tous ces points, le contrôle de la Federal Election Commission (FEC) est essentiel ${ }^{44}$, afin de garantir la transparence sur l'origine et l'emploi des sommes en jeu. Voilà des batailles à venir dont l'issue n'est rien moins que la position majoritaire, ou minoritaire, à la Chambre à Washington et la possibilité pour le président des ÉtatsUnis d'avoir, ou pas, une majorité législative.

\section{Conclusion}

Si les Démocrates ont remporté l'élection présidentielle de l'automne 2020, les résultats, analysés dans le détail, montrent que la victoire est territorialement fragile. Des résultats serrés, voire très serrés, dans des États pivots tels que le Michigan, le Wisconsin, la Pennsylvanie ou la Géorgie doivent les conduire à réfléchir aux conséquences à court et moyen terme pour les prochaines échéances électorales. Audelà du personnage "Trump ", ses électeurs et les difficultés auxquelles ils font face sont une réalité bien ancrée. Selon les projections statistiques issues des résultats des recensements de 2010 et 2020, les perspectives de victoire pour les Démocrates semblent assez difficiles pour la prochaine échéance législative de 2022 (Burmila, 2009), et peut-être aussi pour les échéances suivantes, s'ils ne parviennent pas à prendre en compte certaines tendances à l'œuvre. Le déclin de la population du Nord-Est et du Nord du Midwest se poursuit, ce qui se traduit par une augmentation plus rapide de la population dans la Sunbelt, qui vote plus franchement républicain. Le sud des États-Unis va donc continuer d'être un enjeu majeur pour les élections présidentielles à venir. La Floride reste un État pivot très clivé, comme nous l'avons vu, et un État déterminant, comme il l'a été immanquablement depuis près de 20 ans dans chaque élection présidentielle. Les effets conjugués d'un système électoral qui accentue les écarts, de transformations économiques et sociales, mais aussi culturelles et identitaires, ont conduit à une augmentation de la polarisation politique. Cette polarisation produit des poches bleues ou rouges, à première vue relativement homogènes. Les cartes détaillées des résultats électoraux montrent que cette homogénéité peut être trompeuse. Par ailleurs, l'année 2020, année du recensement décennal, ravive une question historique et politique lancinante : des découpages électoraux contestables pour les élections à la Chambre des représentants. Comme le montre l'illustration 12, publiée le 26 avril par le Census Bureau, des redécoupages devront avoir lieu dans 13 États. Parmi ceux-ci, 5 d'entre eux étaient des États pivots lors des dernières élections (la Floride, la Caroline du Nord, le Michigan, l'Ohio et la Pennsylvanie). Par ailleurs, le Texas, qui n'est pas un état pivot mais qui a une histoire encore récente de découpages et redécoupages contestés, devra créer deux nouveaux $\mathrm{CDs}^{45}$. Les batailles autour des cartes, dans chacun de ces États, ne vont pas manquer. 
Illustration 12 - États qui gagnent ou perdent des représentants à la Chambre des représentants à Washington suite au recensement de 2020

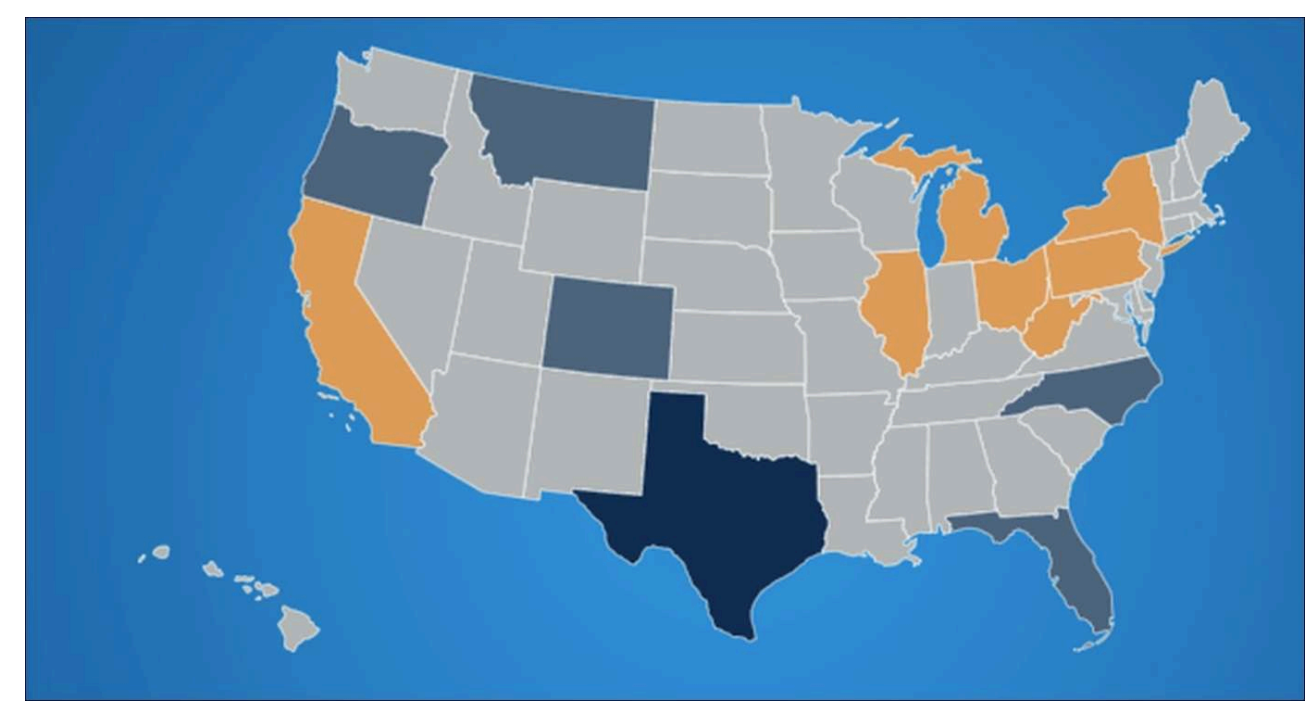

Orange : États qui perdent un représentant;

Gris foncé : États qui gagnent un représentant,

Gris clair : États où il n'y a pas de changement ;

Bleu foncé : État qui gagne deux représentants.

Source : Bureau of the Census, https://www.census.gov/library/visualizations/interactive/historicalapportionment-data-map.htm

\section{BIBLIOGRAPHIE}

Abramowitz A.I., 2010. The Disappearing Center: Engaged Citizens, Polarization, and American Democracy. New Haven, Yale University Press.

Achen C., Bartels L., 2017. Democracy for Realists: Why Elections Do Not Produce Responsive Government. Princeton, Princeton University Press.

Barker D., Carman C., 2012. Representing Red and Blue: How the Culture Wars Change the Way Citizens Speak and Politicians Listen. Oxford, Oxford University Press.

Bartels L., 2018. Partisanship in the Trump Era. The Journal of Politics, vol. 80, n 4, p. 1483-1494.

Burmila E., 2009. The Electoral College after Census 2010 and 2020: The Political Impact of Population Growth and Redistribution. Perspectives on Politics, vol. 7, $n^{\circ} 4$, p. 837-847.

BurnhamW.D., 1970. Critical Elections and the Mainsprings of American Politics. Norton.

Case A., Deaton A., 2020. Deaths of Despair and the Future of Capitalism. Princeton, Princeton University Press.

Foley E., 2020. Presidential Elections and Majority Rule: The Rise, Demise, and Potential Restoration of the Jeffersonian Electoral College. New York, Oxford University Press.

Key V.O., 1955. A Theory of Critical Elections. Journal of Politics, vol. 17, n 1, p. 3-18. 
Keyssar A., 2020. Why Do We Still Have the Electoral College? Cumberland, Harvard University Press.

Lowenthal A.S., 2019. The Ills of Gerrymandering and Independent Redistricting Commissions as the Solution. Harvard Journal on Legislation, vol. 56, $\mathrm{n}^{\circ}$ 1, p. 1-21.

Rankin W., 2016. After the Map. Chicago, Chicago University Press.

Schattschneider E.E., 1960. The Semi-Sovereign People. A Realist's View of Democracy in America. Holt, Rhinehart \& Winston.

Schweber H., 2016. The Limits of Political Representation. American Political Science Review, vol. 110, $\mathrm{n}^{\circ} 2$, p. 382-396.

Sundquist J.L., 1983 (1973). Dynamics of the Party System. Alignment and Realignment of Political Parties in the United States. Brookings.

Turner M.L., LaMacchia R.A., 1999. The US Census, Redistricting and Technology. A 30-Year Perspective. Social Science Computer Review, vol. 17, n 1, pp. 16-26.

\section{NOTES}

1. «For much of the night, the map was a sea of indecision, with state after state hanging in the balance as the votes were tallied at what sometimes seemed like an excruciatingly slow pace ", Dan Balz, 2020. A Stomach-Churning Night of Counting Leaves the Outcome in the Balance. Washington Post, 4 novembre 2020. URL: https://www.washingtonpost.com/politics/a-stomachchurning-night-of-counting-leaves-the-outcome-in-the-balance/ 2020/11/04/2b8f4c60-1e47-11eb_90dd-abd0f7086a91_story

2. Depuis la fin des années 1960, le taux de participation lors de l'élection présidentielle aux États-Unis ne cesse de baisser et est passé de près de 70\% en 1968 à moins de 60\% en 2016. On peut consulter les données collectées sur le temps long par le Bureau of the Census. URL: https:// www.census.gov/library/visualizations/time-series/demo/voting-historical-time-series.html (consulté le 12 avril 2021)

3. Brett Samuels, 2020. Trump Escalates Fight against Mail-in Voting. The Hill, 23 mai 2020. URL: https://thehill.com/homenews/administration/499269-trump-escalates-fight-against-mail-invoting (consulté le 12 avril 2021)

Jane C. Timm, 2020. An All-Out War over Mail Voting has Erupted in Courts Across the US. Here's What's at Stake. NBC News, 15 août 2020. URL: https://www.npr.org/2020/08/28/906676695/ ignoring-fbi-and-fellow-republicans-trump-continues-assault-on-mail-in-voting (consulté le 12 avril 2021)

Miles Parks, 2020. Ignoring FBI and Fellow Republicans, Trump Continues Assault on Mail-In Voting. NPR, 20 août 2020. URL: https://www.npr.org/2020/08/28/906676695/ignoring-fbi-andfellow-republicans-trump-continues-assault-on-mail-in-voting (consulté le 12 avril 2021)

4. Le CARES Act (Coronavirus Aid, Relief, and Economic Security Act) a été adopté le 27 mars 2020 afin de mettre en place des mesures d'aide financières pour soutenir l'emploi et l'activité économique de la population américaine face aux effets sanitaires, économiques, donc, et sociaux de la pandémie. URL: https://home.treasury.gov/policy-issues/cares (consulté le 12 avril 2021)

5. "The things they [Democrats ndlr] had in there [the increased funding for absentee and vote-by-mail options ndlr] were crazy. They had things levels - of voting that, if you ever agreed to it, you'd never have a Republican elected in this country again", Aaron Blake, 2020. Trump Just Comes Out and Says It: The GOP is Hurt When It's Easier to Vote. Washington Post, 30 mars 2020. URL: https:// www.washingtonpost.com/politics/2020/03/30/trump-voting-republicans/ (consulté le 12 avril 2021) 
6. En 2016, le candidat Républicain (Trump) obtient $46 \%$ des voix contre $48,1 \%$ des voix pour la candidate démocrate. On rappellera qu'en 2000, un scénario similaire s'était joué puisque Al Gore (Démocrate) avait obtenu 48,4\% des voix contre $47,9 \%$ pour George W. Bush. Bush avait été élu suite à l'arrêt rendu par la Cour Suprême Bush v. Gore. Cet arrêt tranchait la question de l'irrégularité et de l'attribution de certains bulletins de vote en Floride, état pivot s'il en est. À des fins de comparaison, on peut également trouver des données statistiques depuis la deuxième moitié du $19^{{ }^{e ̀ m e}}$ sur Statista. URL: https://www.statista.com/statistics/1035521/popular-votesrepublican-democratic-parties-since-1828/ (consulté le 12 avril 2021)

7. «The states are only the arbitrary geographic divisions that determine how these individual preferences are distributed across the nation. Optimal electoral reform should make the national expression of those accumulated preferences its proper goal through a popular vote in that single national constituency, the United States of America », Jack Rakove, 2020. Perspectives on Politics, décembre 2020, p. 1217. On peut aussi se référer, par exemple, à un podcast d'octobre 2020 dans lequel Rakove (Stanford University) replace le fonctionnement du Collège Électoral dans une perspective historique remontant aux compromis constitutionnels de la fin du $18^{\text {ème }}$ siècle. URL: https://law.stanford.edu/stanford-legal-on-siriusxm/what-is-the-electoral-collegeand-is-it-fair-with-jack-rakove/ (consulté le 12 avril 2021)

8. On pourra consulter, pour une analyse portant sur "la disparition du centre ", l'ouvrage d'Alan I. Abramowitz, 2010.

9. On pourra se référer à l'article pionnier de Key, 1955; puis aux travaux de E. E. Schattschneider (1960), James L. Sundquist, (1983 et Walter D. Burnham (1970).

10. L'enregistrement de la conversation téléphonique entre Donald Trump et Brad Raffensberger, en charge de la supervision du processus électoral en Géorgie, a été publié début janvier 2021. Au cours de cet échange, Donald Trump fait pression sur son interlocuteur pour que lui soient attribuées 11779 voix supplémentaires. Voir : Transcript : President Trump's Phone Call with Georgia Election Officials, New York Times, 3 janvier 2021. URL: https://www.nytimes.com/ 2021/01/03/us/politics/trump-raffensperger-georgia-call-transcript.html (Consulté le 12 avril 2021)

11. Tous les résultats chiffrés, nationalement, et État par État, sont issus du site parlementaire CSpan. URL: https://www.c-span.org/election/ (Consulté le 12 avril 2021)

12. Voir, par exemple :

Mimi Dwyer \& Joseph Tanfani, 2020. Trump and Biden Protesters Duel Outside Vote-Counting Centers in Cliffhanger Election. Reuters, 4 novembre 2020. URL: https://www.reuters.com/ article/uk-usa-election-protests/trump-and-biden-protesters-duel-outside-vote-countingcenters-in-cliffhanger-election-idUSKBN27LOL7

Joan E Greve, 2020. Race Too Close to Call after Polls Close - as it Happened. The Guardian, 4 novembre 2020. URL: https://www.theguardian.com/us-news/live/2020/nov/03/uselection-2020-live-updates-president-donald-trump-joe-biden-latest-presidential-election-newspolls-update

Claire Hansen, 2020. Presidential Election Undecided as Trump, Biden, See Tight Races in Key States. US News \& World Report, 4 novembre 2020. URL: https://www.usnews.com/news/elections/ articles/2020-11-04/presidential-election-undecided-as-trump-biden-see-tight-races-in-keystates (Consultés le 12 avril 2021)

13. Voir Bureau of the Census. URL: https://www2.census.gov/geo/pdfs/reference/GARM/ Ch4GARM.pdf

14. Kanwal Syed, Elliott Ramos, Ellie Frymire, Naitian Zhou, 2020. In battleground States, Few Counties Flipped even when States Did. NBCNews, 11 novembre 2020. URL: https:// www.nbcnews.com/politics/2020-election/graphic-battleground-state-turnout-2020-electionn1247337 (Consulté le 12 avril 2021) 
15. On peut se référer également à l'analyse au plus près de certains comtés telle que la présente David Weigel, 2020. The Trailer : The 10 Crucial Counties, Revisited. Washington Post, 11 novembre 2020. URL: https://www.washingtonpost.com/politics/2020/11/10/trailer-10-crucial-countiesrevisited/ (Consulté le 12 avril 2021)

16. Mitchell Ferman, 2020. Donald Trump Made Inroads in South Texas this Year. These Voters Explain Why. The Texas Tribune, 13 novembre 2020. URL: https://www.texastribune.org/ 2020/11/13/south-texas-voters-donald-trump/ (Consulté le 12 avril 2021)

17. Alejandro Majorkas est nommé ministre de la sécurité intérieure (Secretary of Homeland Security). C'est un juriste et homme politique né à Cuba, en tant que ministre en charge de la sécurité intérieure, il aura en charge les dossiers liés à l'immigration et les conflits liés à la frontière. Xavier Becerra a été nommé ministre de la santé (Secretary of Health and Human Services), juriste et homme politique, il est né en Californie de parents mexicains et sera le premier Latino à occuper ce poste. Miguel Cardona sera ministre de l'éducation. Né dans le Connecticut, ses parents sont portoricains. Julissa Reynoso Pantaleon sera la cheffe de cabinet de Jill Biden, la première dame. Elle est dominicaine-américaine.

18. On peut également se reporter à une carte qui montre l'évolution des gains ou pertes de voix entre 2016 et 2020, publiée sur le site de NPR (National Public Radio) : Domenico Montanaro \& Connie Hanzhang Jin, 2020. How Biden Won : Ramping Up the Base and Expanding Margins in the Suburbs. NPR, 18 novembre 2020. URL: https://www.npr.org/2020/11/18/935730100/how-bidenwon-ramping-up-the-base-and-expanding-margins-in-the-suburbs (Consulté le 12 avril 2021)

19. " the crisis has reached such a scale that it is becoming a drag on the economy and a threat to national security" Voir Claire Felter, 2020. The US Opioid Epidemic. Council on Foreign Relations, 16 juillet 2020. URL: https://www.cfr.org/backgrounder/us-opioid-epidemic (Consulté le 12 avril 2021)

20. Voir President's Commission on Opioids, URL: https://www.csis.org/podcasts/take-directed/ presidents-commission-combating-drug-addiction-and-opioid-crisis-offers-blunt. (Consulté le 12 avril 2021). L'oxycodone, un opiacé puissant qui est vendu sous le nom d'OxyContin a été certifié par la Food and Drug Administration en 1995 pour ces vertus anti-douleur. En 2017, l'OxyContin et quelques autres opiacés étaient considérés comme responsables d'environ un tiers des 70237 morts par overdose, un chiffre record en soi.

21. Voir les statistiques du Center for Medicare and Medicaid Services, URL: https:// www.cms.gov/Research-Statistics-Data-and-Systems/Statistics-Trends-and-Reports/

NationalHealthExpendData/NationalHealthAccountsHistorical\#: :text=U.S.

\%20health\%20care\%20spending\%20grew,spending\%20accounted\%20for\%2017.7\%20percent

(Consulté le 12 avril 2021)

22. Le Sénat est renouvelé par tiers tous les deux ans. En 2020, 35 sièges de sénateurs (sur les 100 sièges que compte le Sénat) étaient soumis au vote. Parmi les États "pivots » quatre États devaient compter avec une élection sénatoriale : l'Arizona, le Michigan, la Caroline du Nord, et la Géorgie où, événement rare, les deux sièges de sénateurs étaient en jeu.

23. Nous entendons "granularité » ici au sens de la plus petite entité territoriale mobilisée pour les élections à la Chambre des Représentants, soit le Congressional District.

24. "Whatever the cartographer decides to include [on a map] is only made visible by the exclusion of all the other possible subjects ».

25. US House Election Results. URL: https://www.washingtonpost.com/elections/electionresults/house-2020/

26. Le nombre de députés à la Chambre des Représentants à Washington a été arrêté en 1929 à 435. Voir : Permanent Apportionment Act, 1929. URL: https://history.house.gov

27. Constitution des États-Unis, Art. 1, Section 2.

28. Cette carte qui est la dernière en date (publiée au printemps 2021) montre les contours des États, et à l'intérieur de ceux-ci, les comtés et les Congressional Districts (circonscriptions 
électorales pour les élections législatives à la Chambre des Représentants à Washington) numérotés. Le chiffre donne le nombre de représentants de chaque État à Washington. URL: https://www2.census.gov/geo/maps/cong_dist/uswall/cd117/CD117_US_WallMap.pdf 29. On se réfèrera, en particulier aux arrêts de la Cour Suprême Brown v. Topeka (1954) et Baker v. Carr (1962)

30. À la suite du recensement de 2010, l'Arizona, la Floride, la Géorgie, la Caroline du Sud, le Texas, l'Utah, l'état de Washington, gagnent au moins un siège à la Chambre des Représentants ; alors que l'Illinois, l'Iowa, la Louisiane, le Massachussetts, le Michigan, l'Ohio, le New Jersey, l'état de New York, la Pennsylvanie perdent au moins un député.

Voir NCSL (National Conference of State Legislatures): 2010 Census: Gains and Losses in Congressional Seats. URL: http://www.ncsl.org/research/redistricting/spotlightcensus-2010.aspx

31. Le terme "Gerrymandering" est communément utilisé aux États-Unis pour parler de manipulation cartographique des circonscriptions électorales afin de favoriser (ou défavoriser) telle ou telle partie de la population d'un état ou tel ou tel parti pour assurer, ou faciliter, l'élection d'un candidat de façon déloyale. Le terme est issu du nom du Gouverneur du Massachussets, Elbridge Gerry, qui, en 1812, s'était livré à cette manipulation cartographique débouchant sur une circonscription ayant une forme similaire à celle d'une salamandre.

32. Voir Larry W. Carbaugh \& Robert W. Marx, 1990. The TIGER System: A Census Bureau Innovation Serving Data Analysis. Government Information Quarterly, vol. 7, n 3, p. 285-306. URL: https://www.census.gov/geographies/mapping-files/time-series/geo/tiger-line-file.html

33. Pour le 116 ème Congrès (2018-2020), voir l'URL: https://tigerweb.geo.census.gov/ tigerwebmain/TIGERweb_cd116_current.html

34. On pourra citer, de façon non exhaustive :

www.esri.com (créé en 1969) et plus particulièrement: www.esri.com/en-us/arcgis/products/ mapping

www.azavea.com (créé en 2001), voir particulièrement l'onglet «Elections and Politics »;

www.arcbridge.com (créé en 1994), voir particulièrement l'onglet « Redistricting and Boundary Planning » http://arcbridge.com/solutions/\#redr_div

35. URL: https://democraticredistricting.com/

36. Ministre de la Justice de 2009 à 2015.

37. URL: http://www.redistrictingmajorityproject.com/ ou https://rslc.gop/redmap/

38. " change the system to reflect our better selves and end the practice of drawing our districts so that politicians can pick their voters. Let a bipartisan group do it ». Discours sur l'état de l'Union, 12 janvier 2016. URL: https://obamawhitehouse.archives.gov/the-press-office/ 2016/01/12/remarks-president-barack-obama---prepared-delivery-state-union-address

39. On peut aussi consulter les éléments de l'initiative et de l'engagement de Barack Obama dans la perspective des résultats du recensement de 2020, "The movement for fair maps will determine the course of progress on every issue we care about for the next decade. And we can't wait to begin organizing when the redisctricting process starts in 2021. We need to build this movement from the ground up - right now"; « All on the Line. Redisctricting U », URL: https:// allontheline.org/redistrictingu/

40. "One person. One vote. [...] There is a fundamental structural barrier that prevents progress: rigged electoral maps drawn with surgical precision by politicians to preserve their party's political power and silence the will of the people", Voir le «mission statement» de All On The Line, URL: https://allontheline.org/\#mission

41. Voir NCSL (National Conference for State Legislatures), "Into the Thicket: A Redistricting Starter Kit for Legislative Staff”. URL: http://www.ncsl.org/research/redistricting/into-the-thicket-aredistricting-starter-kit-for-legislative-staff.aspx 
42. Ces commissions peuvent être des commissions actives qui ont effectivement pour rôle de mettre en place le redécoupage électoral de l'état, ou elles peuvent n'être que consultatives et proposer des solutions à la législature de l'État qui conserve la responsabilité du découpage. Pour une analyse État par État des types de commissions instaurées ainsi que de leur composition, voir https://www.ncsl.org/research/redistricting/2009-redistricting-commissions-table.aspx. Voir également Alan S. Lowenthal (2019).

43. Michael P. McDonald, 2007. Regulating Redistricting. PS. Political Science and Politics. Octobre 2007, p. 675-679.

44. «Has Redistricting Affected your Campaign », Federal Election Commission, 24 avril 2012. URL: https://www.fec.gov/updates/has-redistricting-affected-your-campaign/

45. On peut se référer, par exemple, à l'arrêt de la Cour Suprême LULAC v. Perry (League of United Latin American Citizens v. Perry, Governor of Texas) 2006. URL: https:// www.supremecourt.gov/opinions/05pdf/05-204.pdf

\section{RÉSUMÉS}

Partant du scrutin de novembre 2020, cet article examine quelques enjeux de la cartographie des résultats de l'élection présidentielle et des élections législatives aux États-Unis. D'une part, une focale cartographique fine permet de nuancer les résultats à l'échelon de l'état fédéré ou, plus local encore, celui du comté. Ces cartes traduisent, en fait, une grande complexité du tissu électoral. Parallèlement, une perspective historique sur les manipulations (gerrymandering) des cartes des circonscriptions (Congressional Districts) apporte un éclairage sur les derniers résultats des élections au Congrès (Chambre des représentants) ainsi que des pistes de réflexion sur la nature de la représentation que seules les cartes permettent de signifier.

Starting out from the Fall 2020 elections in the United States, this article unpacks a few stakes of the cartography of the presidential and House of Representatives election results. The maps of results at the state level as well as the county level, in particular for swing states, show a nuanced picture which stands witness to the great complexity of the electoral fabric. A brief historical overview of gerrymandering - or map manipulation on racial and partisan grounds - helps understand the results of the congressional elections. Cartography is thus not a tool, only, but a major ally in the preservation of representativeness.

\section{INDEX}

Mots-clés : cartographie, élection, États-Unis, découpage électoral, représentation

Keywords : cartography, election, redistricting, representation, United States

\section{AUTEUR}

\section{CHRISTINE ZUMELLO}

Christine Zumello, christine.zumello@sorbonne-nouvelle.fr, est Professeur à l'Université de la Sorbonne Nouvelle. Elle a récemment publié : 
- Zumello C., 2021. La démocratie à la merci des cartes. Silences, découpages et pouvoir aux ÉtatsUnis. In Bracco D., Genay L., Contre-cartographier le monde. Presses Universitaires de Limoges,

p. 64-78. 